ARTICLE

\title{
Light-induced dimension crossover dictated by excitonic correlations
}

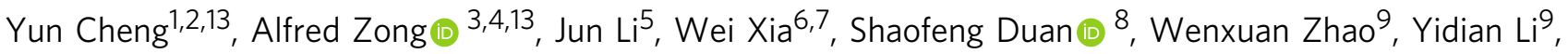

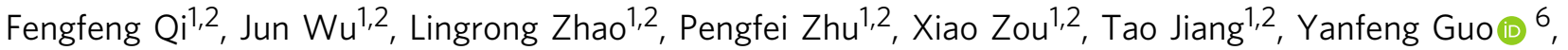 \\ Lexian Yang ${ }^{9}$, Dong Qian (10) ${ }^{8}$, Wentao Zhang (i) ${ }^{8}$, Anshul Kogar (10 ${ }^{10 凶}$, Michael W. Zuerch (1) ${ }^{3,4 \times}$, \\ Dao Xiang (1) $1,2,11,12 \bowtie \&$ Jie Zhang (1) $1,2,11 \bowtie$
}

In low-dimensional systems with strong electronic correlations, the application of an ultrashort laser pulse often yields novel phases that are otherwise inaccessible. The central challenge in understanding such phenomena is to determine how dimensionality and many-body correlations together govern the pathway of a non-adiabatic transition. To this end, we examine a layered compound, $1 T-\mathrm{TiSe}_{2}$, whose three-dimensional charge-density-wave (3D CDW) state also features exciton condensation due to strong electron-hole interactions. We find that photoexcitation suppresses the equilibrium 3D CDW while creating a nonequilibrium 2D CDW. Remarkably, the dimension reduction does not occur unless bound electron-hole pairs are broken. This relation suggests that excitonic correlations maintain the out-of-plane CDW coherence, settling a long-standing debate over their role in the CDW transition. Our findings demonstrate how optical manipulation of electronic interaction enables one to control the dimensionality of a broken-symmetry order, paving the way for realizing other emergent states in strongly correlated systems.

\footnotetext{
${ }^{1}$ Key Laboratory for Laser Plasmas (Ministry of Education), School of Physics and Astronomy, Shanghai Jiao Tong University, Shanghai 200240, China. ${ }^{2}$ Collaborative Innovation Center of IFSA (CICIFSA), Shanghai Jiao Tong University, Shanghai 200240, China. ${ }^{3}$ Department of Chemistry, University of California at Berkeley, Berkeley, CA 94720, USA. ${ }^{4}$ Materials Sciences Division, Lawrence Berkeley National Laboratory, Berkeley, CA 94720, USA. ${ }^{5}$ Beijing National Laboratory for Condensed Matter Physics, Institute of Physics, Chinese Academy of Sciences, Beijing 100190, China. ${ }^{6}$ School of Physical Science and Technology, ShanghaiTech University, Shanghai 201210, China. ${ }^{7}$ ShanghaiTech Laboratory for Topological Physics, Shanghai 201210, China. ${ }^{8}$ Key Laboratory of Artificial Structures and Quantum Control (Ministry of Education), Shenyang National Laboratory for Materials Science, School of Physics and Astronomy, Shanghai Jiao Tong University, Shanghai 200240, China. ${ }^{9}$ State Key Laboratory of Low Dimensional Quantum Physics, Department of Physics, Tsinghua University, Beijing 100084, China. ${ }^{10}$ Department of Physics and Astronomy, University of California at Los Angeles, Los Angeles, CA 90095, USA. ${ }^{11}$ Tsung-Dao Lee Institute, Shanghai Jiao Tong University, Shanghai 200240, China. ${ }^{12}$ Zhangjiang Institute for Advanced Study, Shanghai Jiao Tong

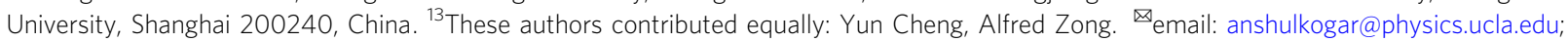
mwz@berkeley.edu; dxiang@sjtu.edu.cn; jzhang1@sjtu.edu.cn
} 
A symmetry-breaking phase transition in $1 \mathrm{D}$ or $2 \mathrm{D}$ is qualitatively distinct from its $3 \mathrm{D}$ counterpart. Strictly speaking, a $1 \mathrm{D}$ chain with short-range interactions cannot undergo a thermodynamic transition at finite temperatures ${ }^{1}$. With short-range interactions in a $2 \mathrm{D}$ plane, a transition can occur through the binding or dissociation of vortex-antivortex pairs $^{2}$. In real materials, however, a more complex situation arises due to nonzero couplings between chains or planes. Although long-range order is possible, significant order parameter fluctuations prevent a mean-field treatment of the transition.

When a system is characterized by both reduced dimensionality and strong electronic correlations, large fluctuations coupled with several competing energy scales provide a fruitful ground for realizing exotic states of matter. This intuition in thermal equilibrium can be leveraged to access new phases using an ultrafast laser pulse, which serves to both modify the Coulomb interaction via carrier screening and to enhance fluctuations when the excited carriers relax $^{3}$. For example, shining a single near-infrared pulse onto the Mott insulating state of $1 T-\mathrm{TaS}_{2}$ produces a "hidden" metallic phase ${ }^{4}$, and illuminating a $\mathrm{PbTiO}_{3} / \mathrm{SrTiO}_{3}$ heterostructure film with an optical pulse leads to a metastable supercrystal ${ }^{5}$. Whereas carrier screening can be well captured by theoretical models to account for such light-induced phenomena ${ }^{5,6}$, out-of-equilibrium fluctuations in low-dimensional systems remain poorly understood due to a lack of experimental probes available to quantify fluctuations along different spatial dimensions. In this article, we study a quasi-2D material with strong electron-hole correlations, $1 T$-TiSe ${ }_{2}$, which also hosts a commensurate CDW. Following femtosecond laser excitation, we observed large, anisotropic CDW fluctuations using $\mathrm{MeV}$ ultrafast electron diffraction (see "Methods" and Supplementary Note 1). Compared to other ultrafast probes, this technique is capable of detecting extremely weak diffuse signals in a momentumresolved manner, unveiling a $3 \mathrm{D}$-to-2D CDW crossover. We show that the transient appearance of the $2 \mathrm{D}$ density wave is controlled by the screening of exciton correlations, providing a unique perspective on the interplay between order parameter fluctuation and electronic correlation in low-dimensional materials.

$1 T-\mathrm{TiSe}_{2}$ has a layered structure where adjacent planes are coupled by van der Waals forces (Fig. 1a). A $3 \mathrm{D}(2 \times 2 \times 2) \mathrm{CDW}$ forms below $T_{c} \approx 195 \mathrm{~K}$, evidenced by the development of the corresponding superlattice peak (Fig. 1b). In monolayer samples or in bulk crystals above $T_{c}$, a $2 \mathrm{D}$ version of the CDW was also reported $^{7-9}$, making it an ideal platform to investigate the morphing dimensions of the symmetry-breaking order. The CDW transition in $1 T-\mathrm{TiSe}_{2}$ is distinguished by concurrent exciton condensation due to the large binding energy between holes in the Se $4 p$ band and electrons in the $\mathrm{Ti} 3 d$ band $^{10}$. However, the exact contribution by the excitonic interaction to the CDW transition has not been clearly identified despite close scrutiny over four decades ${ }^{10-17}$. Here, by correlating the ultrafast

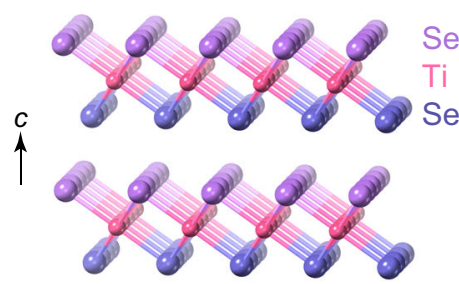

b
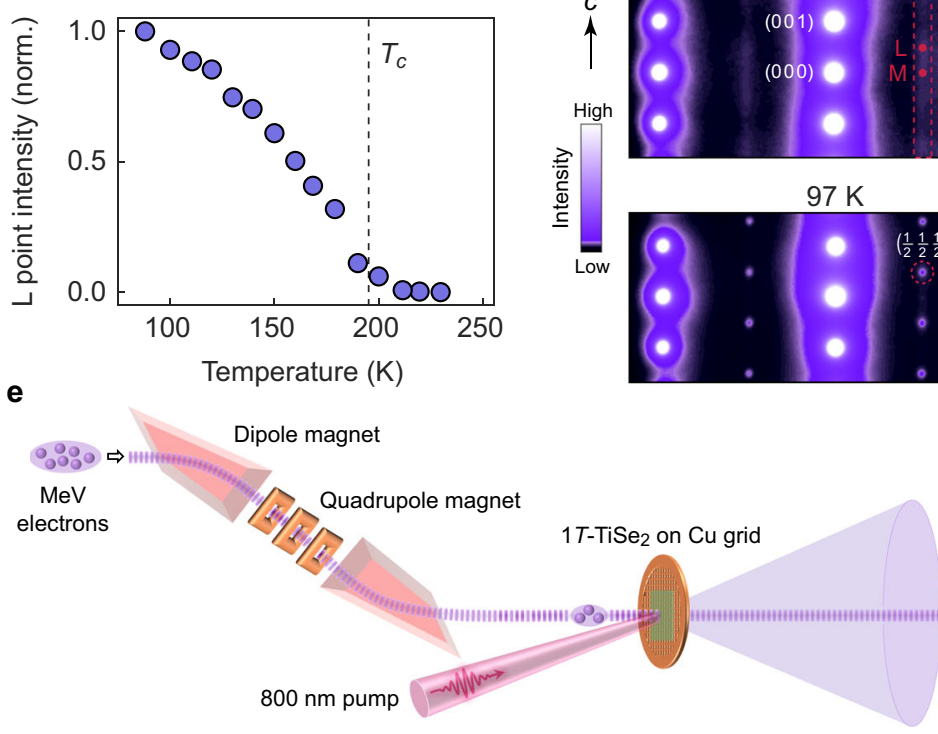

C

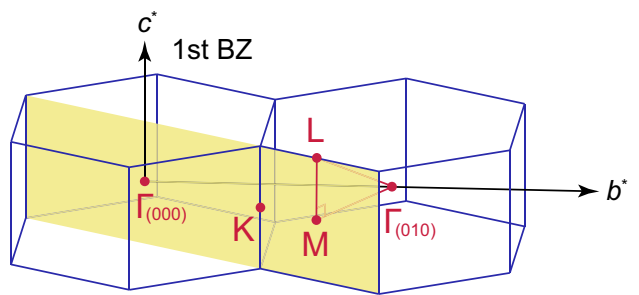

d

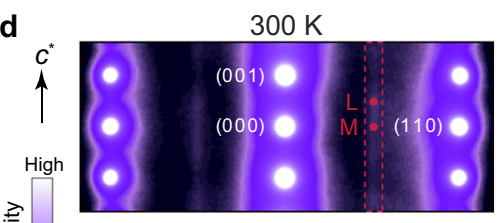

Real space

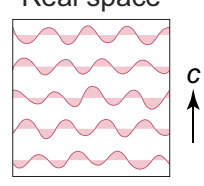

童

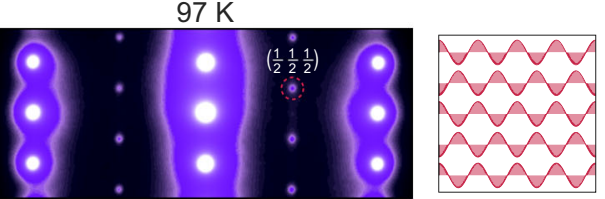

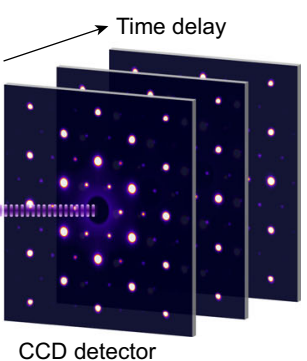

Fig. 1 Equilibrium charge density wave transition in $\mathbf{1 T - T _ { i S e }}$. a Crystal structure of $1 T-\mathrm{TiSe}_{2}$ in the non-CDW state. Layers are bonded by van der Waals forces and each Ti atom is octahedrally coordinated with six Se atoms, where upper and lower Se atoms are colored differently. $\mathbf{b}$ Temperature-dependent intensity evolution of the $\left(\frac{1}{2} \frac{1}{2} \frac{1}{2}\right)$ CDW peak, which is located at the L point in the Brillouin zone (BZ). Intensity is normalized by the value at $88 \mathrm{~K}$. $\mathbf{c}$ Brillouin zones of $1 T-\mathrm{TiSe}_{2}$ with high symmetry points labeled. The yellow plane corresponds to the diffraction plane in (d). d Static electron diffraction patterns in the $\left[1 \overline{1}\right.$ 0] zone axis above and below the CDW transition temperature, $T_{c} \approx 195 \mathrm{~K}$. Diffuse streaks along the $\mathrm{M}-\mathrm{L}$ line at $300 \mathrm{~K}$ (dashed rectangle) is transformed into sharp superlattice peaks at the L point at $97 \mathrm{~K}$ (dashed circle). Schematics on the right show the real space configurations of CDWs in different layers. e Schematic of the ultrafast electron diffraction setup, where a double-bend achromatic lens consisting of a pair of dipole magnets and three quadrupole magnets are used to compress the electron pulse and to reduce the timing jitter (see "Methods"). 
a

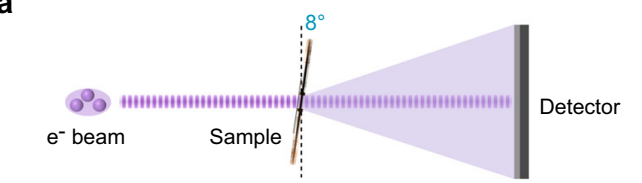

b

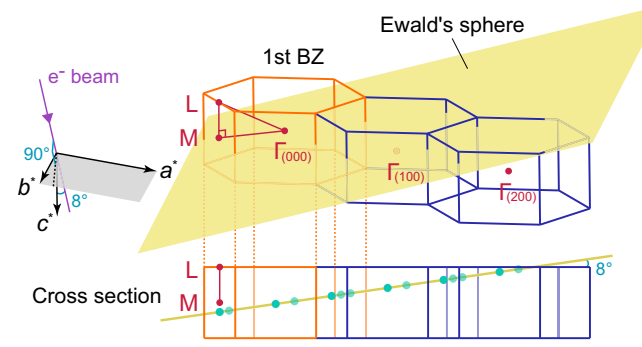

C

\begin{tabular}{|c|c|l}
\hline Label & Diffraction index & \\
\cline { 1 - 2 } 1 & $(0,3,-0.09)$ & \multirow{2}{*}{$\begin{array}{l}\text { Bragg ( } \Gamma \text { point }) \\
\text { M point }\end{array}$} \\
\hline 2 & $(0.5,2,0.05)$ & M \\
\hline 3 & $(-1,0.5,-0.23)$ & Midway between M and L \\
\hline 4 & $(2.5,0.5,0.53)$ & L point \\
\hline
\end{tabular}

d

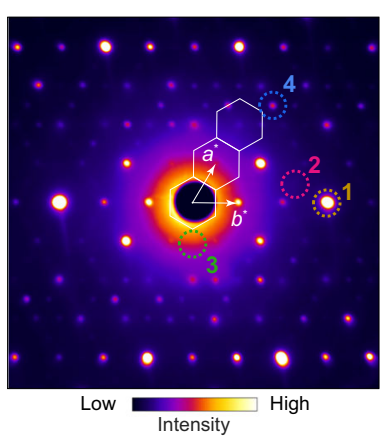

e

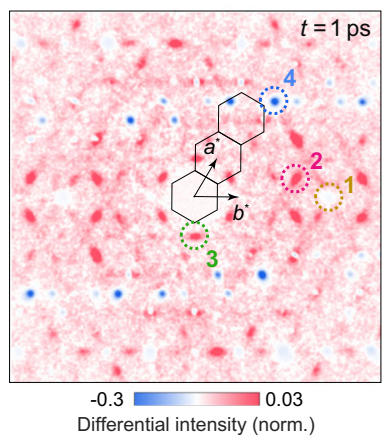

f

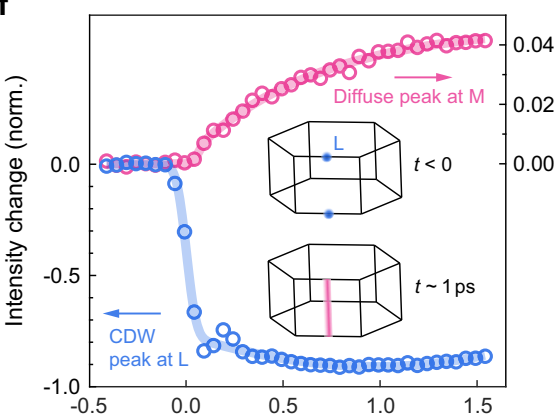

g

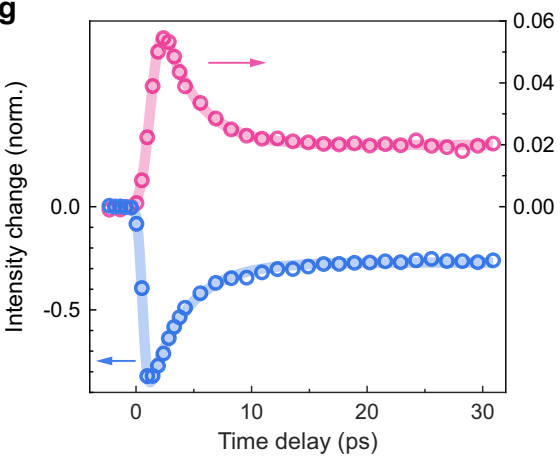

Fig. 2 Light-induced dimension crossover from a 3D to 2D CDW. a Schematic of electron diffraction geometry for data presented in panels (d-g), where an ultrathin sample cleaved in the $\left(\begin{array}{ll}0 & 0\end{array}\right)$ plane is rotated by $8^{\circ}$ relative to the incident electron beam. $\mathbf{b}$ Illustration of the intersection between the reciprocal lattice and the Ewald's sphere, which is locally approximated by a plane that corresponds to the diffraction pattern in (d) and (e). Green dots in the cross-sectional view mark the intersection points between the L-M-L line and the Ewald's sphere. c Miller indices for four color-coded points in the diffraction images. $\mathbf{d}$ Static electron diffraction pattern taken at $88 \mathrm{~K}$ in the tilted geometry with zone axis equal to ( $\overline{36} 5164)$. The three hexagons correspond to the projection of the three Brillouin zones in (b) onto the Ewald's sphere. e Differential diffraction pattern at 1 ps after photoexcitation by an 800-nm, 30-fs pulse. f Time evolution of the CDW peak at the L point (peak 4, blue) and the diffuse peak at the M point (peak 2, red). Intensity values are normalized by their respective averages before photoexcitation. See Supplementary Fig. $5 b$ for traces of additional peaks. Inset illustrates the photoinduced change in the CDW dimensionality, sketched in the reciprocal space. $\mathbf{g}$ The same as (f) but plotted for extended pump-probe time delay, showing the partial recovery to a quasi-equilibrium plateau. $\ln \mathbf{f}$ and $\mathbf{g}$, error bars of intensity change are smaller than the marker size; solid curves are fits to an error function multiplied by an exponential function. The incident fluence for data presented in $(\mathbf{e}-\mathbf{g})$ is $560 \mu \mathrm{J} / \mathrm{cm}^{2}$.

response of bound electron-hole pairs to the CDW fluctuations, we show that excitonic interactions are primarily responsible for the out-of-plane coherence of the CDW. Our work hence offers important insight into the role of electronic correlations in driving the symmetry-breaking transition.

\section{Results}

We first characterize the equilibrium 3D CDW using a transmission electron microscope (TEM). The unit cell doubling in the out-of-plane axis leads to superlattice peaks that, to the first order, only appear at the $\mathrm{L}$ point instead of the $\mathrm{M}$ point in reciprocal space (Fig. 1c), which are verified in our diffraction pattern when electrons are incident along the $\left[\begin{array}{lll}1 & 1 & 0\end{array}\right]$ zone axis. A cut in this plane covers both $\mathrm{L}$ and $\mathrm{M}$ momenta values (Fig. 1d). As the sample is heated above $T_{c}$, the sharp peak at $\mathrm{L}$ morphs into an elongated streak along the $\mathrm{M}-\mathrm{L}$ line. From the Fourier transform relation, these streaks correspond to short-range CDWs within each plane that lack any out-of-plane order. Our temperature-dependent measurements establish that diffraction intensities at different locations along the $\mathrm{M}-\mathrm{L}$ line are a sensitive probe of the CDW dimensionality: A sharp peak solely at $\mathrm{L}$ represents a $3 \mathrm{D}(2 \times 2 \times 2) \mathrm{CDW}$ while diffuse intensity from $\mathrm{M}$ to $\mathrm{L}$ indicates a $2 \mathrm{D}(2 \times 2)$ order without interplane coherence (see schematics in Fig. 1d).

We exploit these signatures of the CDW dimension to study its evolution after photoexcitation. Unlike a standard TEM, the timeresolved setup cannot focus electrons very tightly to minimize temporal pulse broadening. Hence, ultrathin samples with large lateral dimensions are required ${ }^{18}$, obtained by exfoliating a bulk crystal that naturally cleaves in the $\left(\begin{array}{lll}0 & 0 & 1\end{array}\right)$ plane (see "Methods"). To measure the $\mathrm{M}-\mathrm{L}$ line, we tilt the exfoliated flake by $8^{\circ}$ relative to the incoming electron beam (Fig. 2a). In this way, the Ewald's sphere intersects the M-L line at different locations in different Brillouin zones, enabling us to sample the intensity distribution from $\mathrm{M}$ to $\mathrm{L}$ (green dots in Fig. 2b). The static diffraction at $88 \mathrm{~K}$ in this tilted geometry is shown in Fig. 2d, where several momenta between $\mathrm{M}$ and $\mathrm{L}$ are identified (Fig. 2c). As expected from a 3D long-range CDW, superlattice peaks only appear at $\mathrm{L}$ while absent at other locations along the M-L cut, in excellent agreement with the simulated pattern (Supplementary Fig. 2 and Supplementary Note 2).

The photoinduced change at $88 \mathrm{~K}$ following an $800-\mathrm{nm}, 30-\mathrm{fs}$ pulse is presented in the differential intensity plot in Fig. 2e, where we subtract the diffraction pattern before photoexcitation from the one at 1 ps delay. Here, we focus on the fast CDW dynamics along the $\mathrm{M}-\mathrm{L}$ line, during which Debye-Waller heating of the lattice has negligible contribution (see Supplementary Note 3 ). Along the $\mathrm{M}-\mathrm{L}$ cut, a transient intensity decrease is seen at $\mathrm{L}$ (location 4) while enhancements are observed elsewhere (locations 2 and 3), leading to clear diffuse peaks shown in red. To understand the contrasting changes, we examine their timescales by plotting the intensity evolution at $\mathrm{L}$ and $\mathrm{M}$ (Fig. 2f and Supplementary Fig. 5b). There is a clear separation of the initial response times: The intensity drop at $\mathrm{L}$ is completed within $200 \mathrm{fs}$ while the peak growth at $\mathrm{M}$ takes more than 1 ps. The time trace of the CDW peak at $\mathrm{L}$ also 
displays a strongly damped oscillation (blue circles in Fig. $2 \mathrm{f}$ and Supplementary Fig. 5b) that is commonly observed in the ultrafast melting of a charge-ordered state ${ }^{19-21}$, whereas no such oscillation is detected in the diffuse peak at $\mathrm{M}$ (red circles in Fig. 2f). These differences between the $L$ and $M$ points are reminiscent of the timescale dichotomy in the transient creation of a competing CDW in $\mathrm{LaTe}_{3}{ }^{22}$ and during the hidden state formation in $1 T-\mathrm{TaS}_{2}{ }^{23}$, suggesting that the destruction of the $3 \mathrm{D} \mathrm{CDW}$ is driven by a coherent displacive mechanism while 2D CDW fluctuations develop in a spatially inhomogeneous fashion.

Taken together, the intensity evolution along the $\mathrm{M}-\mathrm{L}$ line reveals a dimension crossover from a $3 \mathrm{D}$ to $2 \mathrm{D} \mathrm{CDW}$, signified by a transformation of the sharp peak at $\mathrm{L}$ into a continuous streak stretching the entire $\mathrm{M}-\mathrm{L}$ cut (inset in Fig. $2 \mathrm{f}$ ). The extent of the dimension crossover can be inferred from a comparison of the absolute intensity among different points along the $\mathrm{M}-\mathrm{L}$ line (Supplementary Fig. 6 and Supplementary Note 5): For the fluence corresponding to Fig. 2, photoexcitation yields a transient 2D CDW with nearly no phase coherence between neighboring layers. At long pump-probe delay, the partial recovery to the original 3D CDW is marked by the same relaxation timescale in both $\mathrm{M}$ and $\mathrm{L}$ peaks when some out-of-plane coherence is re-gained (Fig. 2g). After $10 \mathrm{ps}$, the system enters a quasi-equilibrium state, which takes more than $\sim 2 \mathrm{~ns}$ to relax to the pre-excitation configuration (Supplementary Fig. 4b and Supplementary Note 4). The slow recovery of the long-range $3 \mathrm{D} \mathrm{CDW}$ may be attributed to the presence of photoinduced defects ${ }^{24,25}$, which disrupt the CDW phase coherence and account for the persistent broadening of the superlattice peak (Supplementary Fig. 4c).

The light-induced 2D CDW is only observed below the equilibrium $T_{c}$. Above $T_{c}$, photoexcitation only serves to transiently reduce the equilibrium fluctuations. This contrast is best illustrated by the differential diffraction patterns along the $\left[\begin{array}{lll}0 & 0 & 1\end{array}\right]$ zone axis, measured below and above $T_{c}$ under the same photoexcitation conditions (Fig. 3 and Supplementary Fig. 8). At $250 \mathrm{~K}$, the intensity reduction at $\mathrm{M}$ (Fig. $3 \mathrm{~b}$ and Supplementary Fig. $8 \mathrm{~b}$ ) forms a hexagonal pattern with intensities determined by the geometric structure factor ${ }^{26}$. This intensity decrease indicates a rapid suppression of the amplitude of the $2 \mathrm{D}$ density waves within 0.2 ps (Fig. $3 \mathrm{c}$ and Supplementary Note 6). Its stark contrast to the slower intensity enhancement below $T_{c}$ (Fig. 3a, c and Supplementary Fig. 8a, c) suggests that the photoinduced destruction of a $3 \mathrm{D} \mathrm{CDW}$ in a quasi-2D environment is manifested by a dimension crossover that preserves the in-plane order.

One is tempted to interpret the photoinduced crossover from the perspective of the equilibrium transition, where fluctuations of the CDW order reach a maximum at $T_{c}{ }^{27}$. If carrier excitation were to produce similar effects to thermal heating, one would expect transient enhancement of diffuse scattering along $\mathrm{M}-\mathrm{L}$ at temperatures below $T_{c}$ but transient suppression instead above $T_{c}$, consistent with Fig. $3 \mathrm{a}$, b. However, such an analogy to the equilibrium transition breaks down when we investigate the fluence dependence of the nonequilibrium crossover. As the incident fluence is increased, the fast suppression and slow enhancement at $\mathrm{L}$ and $\mathrm{M}$, respectively, become more pronounced (Fig. 4a, b). For a quantitative comparison, we track the intensity changes at $M$ and $\mathrm{L}$ as a function of fluence while fixing the delay time, $t$, at which the photoinduced change is maximal during the ultrafast evolution (Fig. 4c, d). As the maximum of the intensity change at $M$ shifts between 0.3 and 0.7 ps upon increasing the fluence (Fig. 4a), we use the average time delay at $t=0.5 \mathrm{ps}$ in Fig. $4 \mathrm{c}$. The most striking feature in Fig. $4 \mathrm{c}$ is the existence of a fluence threshold at $F_{c} \approx 50 \mu \mathrm{J} / \mathrm{cm}^{2}$, below which negligible intensity enhancement at $\mathrm{M}$ is observed. By contrast, no anomaly is observed across $F_{c}$ at the $\mathrm{L}$ point (Fig. $4 \mathrm{~d}$ ). To confirm the reproducibility of this fluence
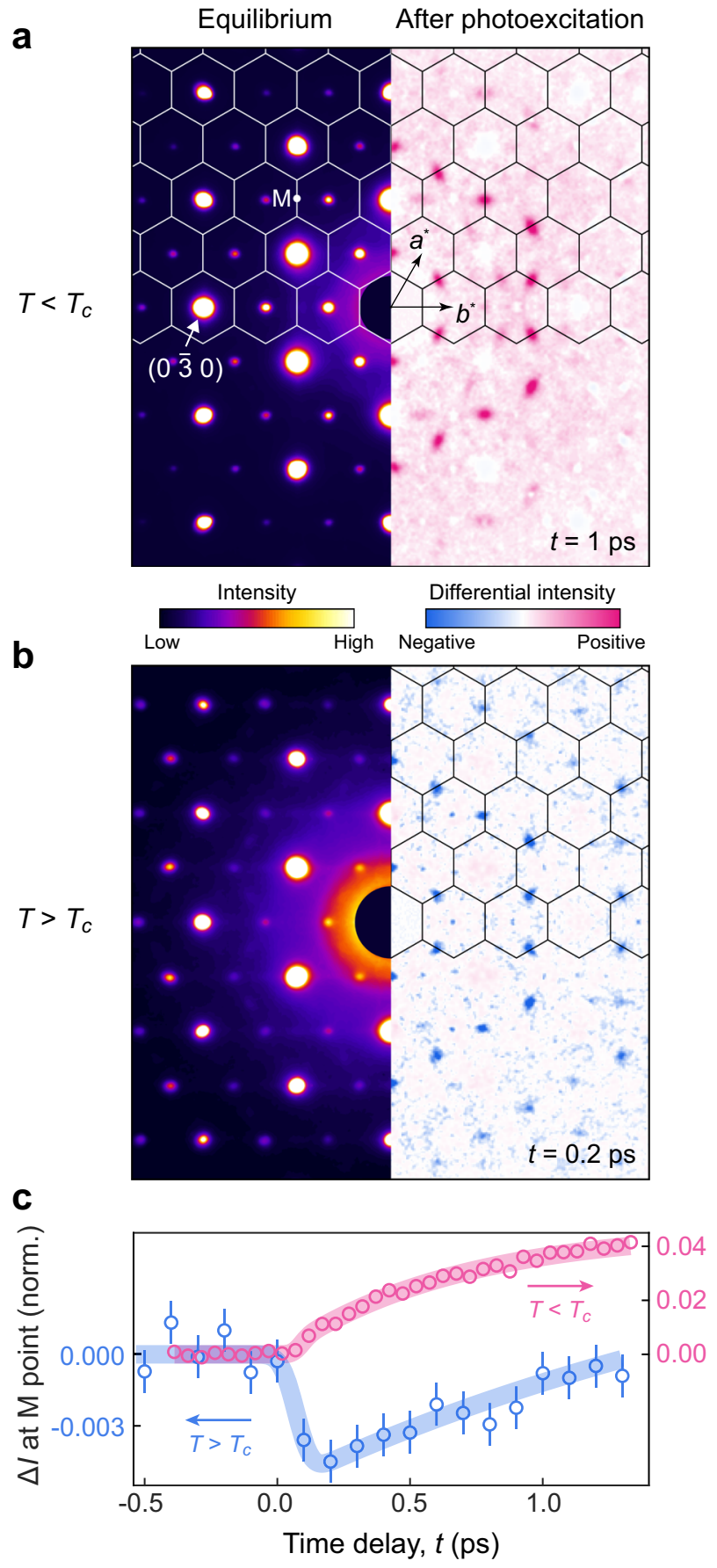

Fig. 3 Distinct photoinduced changes of 2D CDW below and above $\boldsymbol{T}_{\boldsymbol{c}^{\text {}}}$. a, b Left: static electron diffraction patterns along the [0 01 1] zone axis taken at $88 \mathrm{~K}$ (a) and $295 \mathrm{~K}(\mathbf{b})$. Hexagons denote the 2D projection of the Brillouin zones. Right: photoinduced change in the diffraction intensity at 1 ps pump-probe delay at $88 \mathrm{~K}$ (a) and at $0.2 \mathrm{ps}$ delay at $250 \mathrm{~K}$ (b). The different time delays are chosen to reflect the distinct timescales of the intensity change at different temperatures (see panel c). All diffraction images were symmetrized for enhanced statistics. The equilibrium patterns share the same color scale while that of the differential patterns is individually adjusted to highlight the feature at the $M$ point. See Supplementary Fig. 8 for a side-by-side comparison of differential patterns at two time delays with the same color scale. c Temporal evolutions of intensity change at the $\mathrm{M}$ point at $88 \mathrm{~K}$ (red) and $250 \mathrm{~K}$ (blue). Intensities are normalized to respective pre-excitation values. Error bars, if larger than the marker size, represent the standard deviation of intensity values prior to excitation. The incident fluence for all panels is $560 \mu \mathrm{J} / \mathrm{cm}^{2}$. 
a

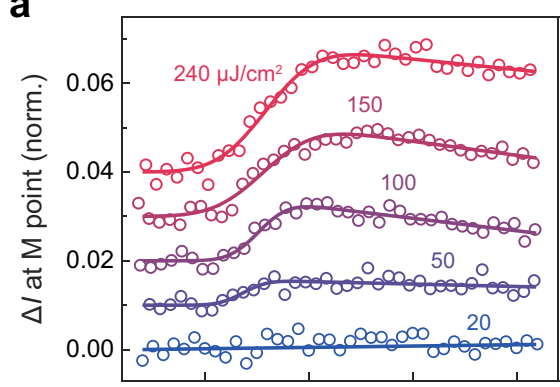

b

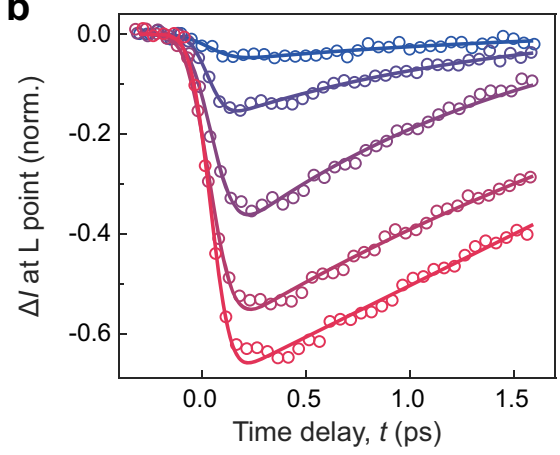

C

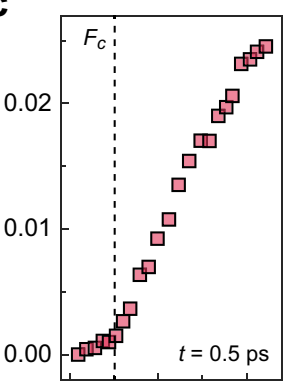

d

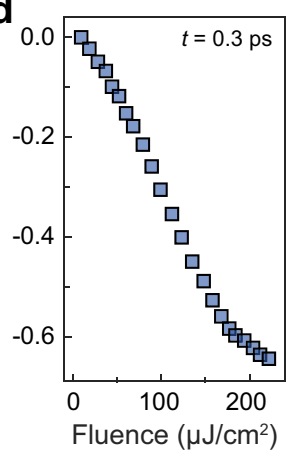

e

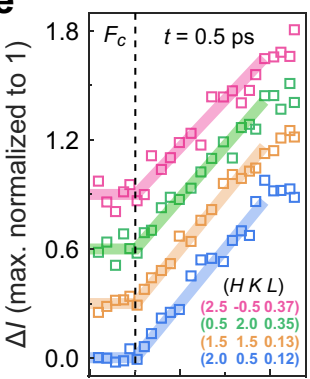

f

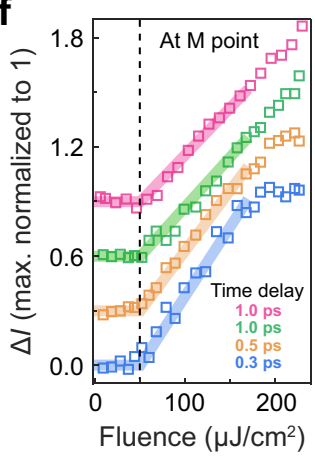

Fig. 4 Fluence-dependent dimension crossover of the CDW. a, b Time evolution of changes in integrated intensities $(\Delta /)$ at $M$ and $L$ points, measured at $88 \mathrm{~K}$. Intensity values are normalized by the averages before photoexcitation. Incident fluences are labeled and color-coded. Solid curves are fits to an error function multiplied by an exponential relaxation and serve as a guide to the eye. For the $\mathrm{M}$ point, multiple diffuse peaks are used for enhanced signal-to-noise ratio. For the $\mathrm{L}$ point, superlattice peak $\left(2 \frac{1}{2} \frac{1}{2}\right)$ is used. c, d Fluence-dependent changes in intensity at the M point at 0.5 ps time delay (c) and at the L point at 0.3 ps time delay (d). The vertical dashed line marks the critical fluence, $F_{c,}$ above which the photoinduced intensity at the $M$ point starts to rapidly increase. A separate critical fluence is observed in the $L$ point intensity at long time delay (see Supplementary Fig. 4 and Supplementary Note 4). e Fluence-dependent intensity change at various momenta along the $\mathrm{M}-\mathrm{L}$ cut, measured at a fixed time delay of $0.5 \mathrm{ps}$. $\mathbf{f}$ Fluence-dependent intensity change at various pump-probe delays, measured at the $\mathrm{M}$ point. Red data points at 1.0 ps were taken from a separate sample compared to the other data points. In $\mathbf{e}$ and $\mathbf{f}$, traces are normalized so that the maximum change is 1 ; the traces are also vertically shifted by 0.3 for clarity. Curves are guides to the eye.

Table 1 Energy threshold for melting the exciton order based on different observables in various pump-probe techniques, expressed in terms of absorbed energy per normal state unit cell (u.c.).

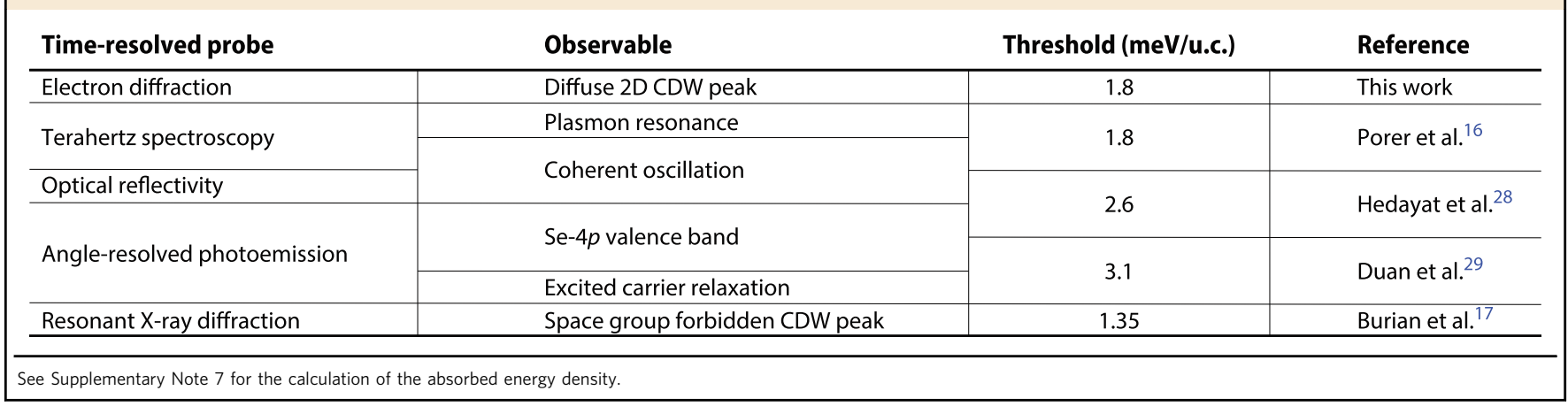

threshold, we plot the fluence-dependent intensity change at other pump-probe delays and other momenta along the M-L cut, measured in different samples (Fig. 4e, f). The same value of $F_{c}$ is observed solely in the diffuse scattering signal away from $\mathrm{L}$ in the $\mathrm{M}-\mathrm{L}$ cut that represents the transient formation of a 2D CDW. This distinction between the $\mathrm{M}$ and $\mathrm{L}$ points suggests that below $F_{c}$, laser pulses weaken the amplitude of the $3 \mathrm{D}$ CDW but the interplane coherence is not affected. Only at fluences above $F_{c}$ does the dimensional crossover start to take place.

\section{Discussion}

The existence of a fluence threshold is unexpected from equilibrium considerations, where a temperature increase towards $T_{c}$ continuously strengthens $\mathrm{CDW}$ fluctuations ${ }^{27}$. As the initial effect of photoexcitation at $1.55 \mathrm{eV}$ is free carrier generation, the fluence threshold hints at a critical carrier density that controls the dimension crossover in the CDW. The observed value of $F_{c}$ is translated into a transient free carrier density of $\sim 4 \times 10^{20} \mathrm{~cm}^{-3}$ (see Supplementary Note 7), which corresponds to a ThomasFermi screening length just short of the superlattice period both in and out-of-plane ${ }^{16}$. Hence, Coulomb attraction between electrons and holes on the length scale of the CDW wavelength is strongly suppressed above $F_{c}$, marking a transient breakdown of the excitonic correlations. The link between $F_{c}$ and the dissociation of bound electron-hole pairs is corroborated by several timeresolved experiments that are sensitive to carrier dynamics (Table 1) $)^{16,17,28,29}$. In time-resolved terahertz spectroscopy, a 
plasmon pole in the energy loss function is significantly modified above $F_{\mathcal{C}}$, suggesting that photoexcitation has transiently melted the exciton condensate ${ }^{16}$. This conclusion is cross-validated by femtosecond resonant X-ray diffraction. At $F_{c}$, there is a complete suppression of a special type of superlattice peaks ${ }^{17}$, which are space-group forbidden and can only be observed if excitonic correlations are present. Time-resolved optical reflectivity and photoemission also demonstrate a similar threshold at $F_{c}$ in the valence band shift and carrier relaxation rate, both of which are sensitive to the excitonic order ${ }^{29,30}$. All of these observations point towards a critical free carrier density at $F_{c}$, above which excitonic correlations transiently disappear.

Such interpretation of the fluence threshold suggests that the interlayer CDW coherence persists unless the exciton condensate is first melted due to carrier excitation and a screened potential. The chief role of exciton correlation in the CDW transition is thus to maintain the interplane coherence, thanks to the longrange nature of Coulomb coupling between Ti $3 d$ electrons and Se $4 p$ holes. Below the fluence threshold $F_{c}$, the effective excitonic correlation length exceeds twice the interlayer spacing, hence contributing to locking the CDW phase in the out-of-plane direction. Our analysis also offers a natural explanation for why the CDW lattice distortion persists even if excitons are destroyed by photoexcitation ${ }^{16}$ : The non-thermal separation between the lattice and excitonic orders is a manifestation of the evolving CDW dimension.

The investigation of $1 T-\mathrm{TiSe}_{2}$ yields new insights toward the understanding of nonequilibrium phenomena that can arise in low-dimensional materials with strong electronic correlations, from light-induced superconductivity in cuprates ${ }^{31}$ to the insulator-metal transition in $1 T-\mathrm{TaS}_{2}{ }^{4,32}$. Not only does our work establish dimensional crossover as the principal pathway in a non-adiabatic transition, it also demonstrates a viable route to control ordered phases with light through impulsive modification of Coulomb interactions, providing access to novel states of matter that are hidden in thermal equilibrium.

\section{Methods \\ Sample preparation. High-quality single crystals of $1 T$-TiSe $e_{2}$ were grown by chemical vapor transport with an iodine transport agent. Ti and Se were mixed in a molar ratio of 1:2 and placed into an alumina crucible before sealed into a quartz tube. The quartz tube was heated to $700{ }^{\circ} \mathrm{C}$ and $1 T$-TiSe $e_{2}$ crystals were synthesized at the $650{ }^{\circ} \mathrm{C}$ zone for 2 weeks. $1 T$-TiSe $e_{2}$ thin flakes were obtained by repeated exfoliation of the bulk crystal with polydimethylsiloxane films (PDMS, Gel-Pak). Flakes were pre-screened for thickness and uniformity with an optical microscope using the color contrast and further characterized by atomic force microscopy. Selected flakes were detached from PDMS in ethanol and scooped onto a 600 mesh/inch TEM grid. The resulting free-standing flake has a typical lateral dimension of $\sim 300 \mu \mathrm{m}$ and thickness of $\sim 60 \mathrm{~nm}$.}

MeV ultrafast electron diffraction. The schematic of the MeV UED beamline is shown in Fig. 1e. The $800-\mathrm{nm}(1.55-\mathrm{eV}), 30$-fs pulses from a Ti:sapphire regenerative amplifier system operating at a repetition rate of $100 \mathrm{~Hz}$ (Vitara and Legend Elite Duo $\mathrm{HE}$, Coherent) were split into pump and probe branches. The probe branch was frequency tripled in nonlinear crystals before illuminating a photocathode for electron pulse generation. After being accelerated by an intense radio-frequency field to relativistic velocity $(\sim 0.989 c)$, the electron beam went through a double-bend achromatic lens for pulse compression and jitter removal. The typical electron beam spot size on the sample was $\sim 150 \mu \mathrm{m}$ measured in full-width at half maximum (FWHM), nearly five times smaller than the size of the pump pulse, ensuring a homogeneous photoexcitation condition. The temporal delay between the pump and probe pulses was adjusted by a linear translation stage. Diffracted electron beams were incident on a phosphor screen (P43) and the image was collected by an electron-multiplying charge-coupled device. In the low-temperature experiments, the sample was cooled by liquid nitrogen. Further details of the UED beamline can be found in ref. ${ }^{33}$.

Transmission electron microscopy. The TEM experiments were conducted using a JEOL JEM-2100F microscope, where the accelerating voltage was $200 \mathrm{kV}$. The cross-section TEM sample was prepared by a focused-ion beam technique based on the standard lift-out procedure and the sample size was approximately $20 \mu \mathrm{m} \times$ $10 \mu \mathrm{m} \times 60 \mathrm{~nm}$. The beam spot size was $10 \mu \mathrm{m}$ in diameter and the current density was $0.2 \mathrm{pA} / \mathrm{cm}^{2}$. The electron beam was incident on the sample along the direction of a straight height step, which is normally the edge of a low-index plane, and the zone axis was further confirmed by diffraction pattern simulations. Lowtemperature data were collected with liquid nitrogen cooling.

\section{Data availability}

All of the data supporting the conclusions are available within the article and the Supplementary Information. Additional data are available from the corresponding authors upon reasonable request.

Received: 17 August 2021; Accepted: 17 January 2022; Published online: 18 February 2022

\section{References}

1. van Hove, L. Sur L'intégrale de configuration pour les systèmes de particules à une dimension. Physica 16, 137 (1950).

2. Kosterlitz, J. M. \& Thouless, D. J. Ordering, metastability and phase transitions in two-dimensional systems. J. Phys. C: Solid State Phys. 6, 1181 (1973).

3. Sun, Z. \& Millis, A. J. Transient trapping into metastable states in systems with competing orders. Phys. Rev. X 10, 021028 (2020).

4. Stojchevska, L. et al. Ultrafast switching to a stable hidden quantum state in an electronic crystal. Science 344, 177 (2014).

5. Stoica, V. A. et al. Optical creation of a supercrystal with three-dimensional nanoscale periodicity. Nat. Mater. 18, 377 (2019).

6. Karpov, P. \& Brazovskii, S. Modeling of networks and globules of charged domain walls observed in pump and pulse induced states. Sci. Rep. 8, 4043 (2018).

7. Chen, $\mathrm{P}$. et al. Charge density wave transition in single-layer titanium diselenide. Nat. Commun. 6, 8943 (2015).

8. Chen, P. et al. Hidden order and dimensional crossover of the charge density waves in $\mathrm{TiSe}_{2}$. Sci. Rep. 6, 37910 (2016).

9. Watson, M. D. et al. Strong-coupling charge density wave in monolayer $\mathrm{TiSe}_{2}$ 2D Mater. 8, 015004 (2020).

10. Kogar, A. et al. Signatures of exciton condensation in a transition metal dichalcogenide. Science 358, 1314 (2017).

11. Hughes, H. P. Structural distortion in $\mathrm{TiSe}_{2}$ and related materials-a possible Jahn-Teller effect? J. Phys. C: Solid State Phys. 10, L319 (1977).

12. Suzuki, N., Yamamoto, A. \& Motizuki, K. Microscopic theory of the CDW state of $1 T$-TiSe 2 . J. Phys. Soc. Jpn. 54, 4668 (1985).

13. Kidd, T. E., Miller, T., Chou, M. Y. \& Chiang, T.-C. Electron-hole coupling and the charge density wave transition in $\mathrm{TiSe}_{2}$. Phys. Rev. Lett. 88, 226402 (2002).

14. Cercellier, H. et al. Evidence for an excitonic insulator phase in $1 T-\mathrm{TiSe}_{2}$. Phys. Rev. Lett. 99, 146403 (2007).

15. Calandra, M. \& Mauri, F. Charge-density wave and superconducting dome in $\mathrm{TiSe}_{2}$ from electron-phonon interaction. Phys. Rev. Lett. 106, 196406 (2011).

16. Porer, $M$. et al. Non-thermal separation of electronic and structural orders in a persisting charge density wave. Nat. Mater. 13, 857 (2014).

17. Burian, M. et al. Structural involvement in the melting of the charge density wave in $1 T$-TiSe ${ }_{2}$. Phys. Rev. Res. 3, 013128 (2021).

18. Bie, Y.-Q., Zong, A., Wang, X., Jarillo-Herrero, P. \& Gedik, N. A versatile sample fabrication method for ultrafast electron diffraction. Ultramicroscopy 230, 113389 (2021)

19. Huber, T. et al. Coherent structural dynamics of a prototypical chargedensity-wave-to-metal transition. Phys. Rev. Lett. 113, 026401 (2014).

20. Moore, R. G. et al. Ultrafast resonant soft X-ray diffraction dynamics of the charge density wave in $\mathrm{TbTe}_{3}$. Phys. Rev. B 93, 024304 (2016).

21. Wall, S. et al. Ultrafast disordering of vanadium dimers in photoexcited $\mathrm{VO}_{2}$. Science 362, 572 (2018).

22. Zong, A. et al. Role of equilibrium fluctuations in light-induced order. Phys. Rev. Lett. 127, 227401 (2021).

23. Ravnik, J., Vaskivskyi, I., Mertelj, T. \& Mihailovic, D. Real-time observation of the coherent transition to a metastable emergent state in $1 T-\mathrm{TaS}_{2}$. Phys. Rev. B 97, 075304 (2018).

24. Vogelgesang, S. et al. Phase ordering of charge density waves traced by ultrafast low-energy electron diffraction. Nat. Phys. 14, 184 (2018)

25. Zong, A. et al. Evidence for topological defects in a photoinduced phase transition. Nat. Phys. 15, 27 (2019).

26. Otto, M. R. et al. Mechanisms of electron-phonon coupling unraveled in momentum and time: The case of soft phonons in $\mathrm{TiSe}_{2}$. Sci. Adv. 7, eabf2810 (2021).

27. Holt, M., Zschack, P., Hong, H., Chou, M. Y. \& Chiang, T.-C. X-ray studies of phonon softening in $\mathrm{TiSe}_{2}$. Phys. Rev. Lett. 86, 3799 (2001).

28. Hedayat, H. et al. Excitonic and lattice contributions to the charge density wave in $1 T-\mathrm{TiSe}_{2}$ revealed by a phonon bottleneck. Phys. Rev. Res. 1, 023029 (2019). 
29. Duan, S. et al. Optical manipulation of electronic dimensionality in a quantum material. Nature 595, 239 (2021).

30. Hedayat, H. et al. Investigation of the non-equilibrium state of strongly correlated materials by complementary ultrafast spectroscopy techniques. $N$. J. Phys. 23, 033025 (2021).

31. Kaiser, S. Light-induced superconductivity in high- $T_{c}$ cuprates. Phys. Scr. 92, 103001 (2017).

32. Stahl, Q. et al. Collapse of layer dimerization in the photo-induced hidden state of $1 T-\mathrm{TaS}_{2}$. Nat. Commun. 11, 1247 (2020).

33. Qi, F. et al. Breaking 50 femtosecond resolution barrier in $\mathrm{MeV}$ ultrafast electron diffraction with a double bend achromat compressor. Phys. Rev. Lett. 124, 134803 (2020).

\section{Acknowledgements}

We thank Weidong Luo, Chengyang $\mathrm{Xu}$, Sheng Meng, and Yu He for helpful discussions. D.X. and J.Z. acknowledge support from the National Key R\&D Program of China (No. 2021YFA1400202), the National Natural Science Foundation of China (grant No.'s 11925505,11504232 , and 11721091), and the office of Science and Technology, Shanghai Municipal Government (No. 16DZ2260200). J.L. acknowledges support from the National Natural Science Foundation of China under grant No. 12074408. L.Y. acknowledges support from the National Natural Science Foundation of China (grant No. 11774190). W.Z. acknowledges support from the Ministry of Science and Technology of China (2016YFA0300501) and the National Natural Science Foundation of China (11974243), and additional support from a Shanghai talent program. Y.G. acknowledges support from the National Natural Science Foundation of China (grant No. 11874264). D.Q. acknowledges support from the Ministry of Science and Technology of China (grant No. 2016YFA0301003), and the National Natural Science Foundation of China (grant No.'s 12074248 and 11521404). A.Z. acknowledges support from the Miller Institute for Basic Research in Science. M.W.Z. acknowledges funding by the W. M. Keck Foundation, funding from the UC Office of the President within the Multicampus Research Programs and Initiatives (M21PL3263), and the Hellman Fellows Fund.

\section{Author contributions}

D.X. and J.Z. supervised the project. Y.C. and A.Z. designed the research and performed the data analysis with help from A.K., W.Z., and D.X. F.Q., L.Z., P.Z., X.Z., and T.J. constructed and maintained the MeV-UED beamline. Y.C. and J.W. collected the UED data. J.L. per- formed the TEM measurements and made significant contributions to the simulation of the diffraction patterns. W.X. and Y.G grew the single crystals. A.Z. and Y.C. wrote the manuscript with critical input from A.K., M.W.Z., W.Z., D.Q., D.X. and all other authors.

\section{Competing interests}

The authors declare no competing interests.

\section{Additional information}

Supplementary information The online version contains supplementary material available at https://doi.org/10.1038/s41467-022-28309-5.

Correspondence and requests for materials should be addressed to Anshul Kogar, Michael W. Zuerch, Dao Xiang or Jie Zhang.

Peer review information Nature Communications thanks the anonymous reviewers for their contribution to the peer review of this work.

Reprints and permission information is available at http://www.nature.com/reprints

Publisher's note Springer Nature remains neutral with regard to jurisdictional claims in published maps and institutional affiliations.

cc) (i) Open Access This article is licensed under a Creative Commons Attribution 4.0 International License, which permits use, sharing, adaptation, distribution and reproduction in any medium or format, as long as you give appropriate credit to the original author(s) and the source, provide a link to the Creative Commons license, and indicate if changes were made. The images or other third party material in this article are included in the article's Creative Commons license, unless indicated otherwise in a credit line to the material. If material is not included in the article's Creative Commons license and your intended use is not permitted by statutory regulation or exceeds the permitted use, you will need to obtain permission directly from the copyright holder. To view a copy of this license, visit http://creativecommons.org/ licenses/by/4.0/.

(C) The Author(s) 2022 